\title{
ON THE XIAO CONJECTURE FOR PLANE CURVES
}

\author{
F. F. FAVALE, J. C. NARANJO, AND G. P. PIROLA
}

\begin{abstract}
Let $f: S \longrightarrow B$ be a non-trivial fibration from a complex projective smooth surface $S$ to a smooth curve $B$ of genus $b$. Let $c_{f}$ the Clifford index of the general fibre $F$ of $f$. In BGN16] it is proved that the relative irregularity of $f, q_{f}=h^{1,0}(S)-b$ is less or equal than or equal to $g(F)-c_{f}$. In particular this proves the (modified) Xiao's conjecture: $q_{f} \leq \frac{g(F)}{2}+1$ for fibrations of general Clifford index. In this short note we assume that the general fiber of $f$ is a plane curve of degree $d \geq 5$ and we prove that $q_{f} \leq g(F)-c_{f}-1$. In particular we obtain the conjecture for families of quintic plane curves. This theorem is implied for the following result on infinitesimal deformations: let $F$ a smooth plane curve of degree $d \geq 5$ and let $\xi$ be an infinitesimal deformation of $F$ preserving the planarity of the curve. Then the rank of the cup-product map $H^{0}\left(F, \omega_{F}\right) \stackrel{\cdot \xi}{\longrightarrow} H^{1}\left(F, O_{F}\right)$ is at least $d-3$. We also show that this bound is sharp.
\end{abstract}

\section{INTRODUCTION}

Let $f: S \longrightarrow B$ be a non-isotrivial fibration from a complex projective smooth surface $S$ to a smooth curve $B$ of genus $b$. A natural question is trying to understand the relation between the invariants of the surface, the base curve $B$ and of the general fibre $F$. Nonisotrivial means that the smooth fibres are not isomorphic to each other, in other words: the natural modular map $B^{0} \longrightarrow \mathcal{M}_{g}$ in the moduli space of curves of genus $g=g(F)$ is not constant, where $B^{0}$ is the open set of $B$ with smooth fibres. The invariants we consider are the relative irregularity $0 \leq q_{f}:=h^{1,0}(S)-g(B)$ and the genus $g$ of the general fibre. Xiao proved in [X87] that for non-isotrivial fibrations the inequality

$$
q_{f} \leq \frac{5 g+1}{6}
$$

holds, and he formulated in [X88] the conjecture

$$
q_{f} \leq \frac{g+1}{2}
$$

After some counterexamples found by Pirola in [P92] (see also [AP16]) the conjecture is nowadays reformulated as follows

Modified Xiao's conjecture: For non-isotrivial fibrations it holds that

$$
q_{f} \leq \frac{g}{2}+1
$$

Observe that it is equivalent to the initial conjecture for $g$ odd.

2010 Mathematics Subject Classification. 14H50,14D06,14J99,14B10.

The first named author has been partially supported by INdAM - GNSAGA and by FIRB2012 "Moduli spaces and applications", which are granted by MIUR, and by IMUB during his stay at the University of Barcelona; the second named author has been partially supported by the Proyecto de Investigación MTM2015-65361-P; the third named author is partially supported by PRIN 2015 "Moduli spaces and Lie Theory", INdAM - GNSAGA and FAR 2016 (PV) "Varietà algebriche, calcolo algebrico, grafi orientati e topologici". We would like to thank Miguel Ángel Barja, Víctor González-Alonso and Carlos d'Andrea for useful comments. 
There are some evidences for this conjecture: Xiao proved that it holds if $b=0$ and it is known to be true when $F$ is hyperelliptic (see C95]). Moreover in [BGN16] a upper bound of $q_{f}$ is found in terms of the Clifford index $c_{f}$ of the general fibre. Remember that the Clifford index of the curve $F$ is defined as:

$$
\operatorname{Cliff}(F):=\min \left\{\operatorname{deg}(L)-2\left(h^{0}\left(F, \mathcal{O}_{F}(L)\right)-1\right) \mid h^{0}\left(F, \mathcal{O}_{F}(L)\right) \geq 2, h^{1}\left(F, \mathcal{O}_{F}(L)\right) \geq 2\right\} .
$$

Clifford's Theorem states that $\operatorname{Cliff}(F) \geq 0$ and it is 0 if and only if $F$ is hyperelliptic. It is known that $\operatorname{Cliff}(F)=1$ if and only if either $F$ is trigonal or isomorphic to a smooth quintic plane curve. A smooth plane curve of degree $d$ has Clifford index $d-4$ and a general curve in $\mathcal{M}_{g}$ has Clifford index $\left\lfloor\frac{g-1}{2}\right\rfloor$.

The main theorem in [BGN16] says that for a non-isotrivial fibration it holds

$$
q_{f} \leq g-c_{f}
$$

In particular the conjecture is true when the general fiber has general Clifford index. Combining the results given above one easily checks that the conjecture is true for $g \leq 4$ and that the first open case corresponds to $g \geq 5$ and $c_{f}=1$. In this paper we take care of the quintic plane curve case. More generally we consider families of smooth plane curves of degree $d \geq 5$. Our main theorem is the following:

Theorem 1.1. Let $f: S \longrightarrow B$ be a fibration such that the general fibre is a plane curve of degree $d \geq 5$. Then

$$
q_{f} \leq g-c_{f}-1=g-(d-4)-1=g-d+3
$$

In the case $d=5$, hence $g=6$, we obtain $q_{f} \leq 4$ which is the predicted bound. Hence we obtain:

Corollary 1.2. The modified Xiao's conjecture holds for fibrations with general fibre a quintic plane curve.

The idea of the proof of (1.1) is as follows: let us fix a general point of $B$ and let $F$ the fibre at this point, then $f$ induces an infinitesimal deformation $\xi \in H^{1}\left(F, T_{F}\right)$. The kernel $W_{\xi}$ of the cup-product map -

$$
H^{0}\left(F, \omega_{F}\right) \stackrel{\cdot \xi}{\longrightarrow} H^{1}\left(F, \mathcal{O}_{F}\right)
$$

contains the vector space $H^{0}\left(S, \Omega_{S}^{1}\right) / f^{*} H^{0}\left(B, \omega_{B}\right)$ (see [BGN16, Section 2] for the details). Therefore $q_{f} \leq \operatorname{dim} W_{\xi}=g-\operatorname{rank}(\cdot \xi)$. Thus it is enough to find a lower bound of the rank of the map given by the cup-product with $\xi$. Then the next Theorem immediately implies Theorem (1.1):

Theorem 1.3. Let $\xi$ be an infinitesimal deformation of $F$ as smooth plane curve, then the rank of the map $H^{0}\left(F, \omega_{F}\right) \stackrel{\cdot \xi}{\longrightarrow} H^{1}\left(F, \mathcal{O}_{F}\right)$ is at least $d-3$, and this bound is realized for the Fermat curve.

The rest of the paper is devoted to the proof of Theorem (1.3). In the next section we recall how to use the Jacobian ring of a plane curve in order to understand the cohomology of the curve $F$ and the cup-product maps. We also state two theorems of Green on multiplication and restriction maps of polynomials. In section 3 we use these facts combined with the classical Macaulay's Theorem to prove Theorem (1.3). 


\section{Preliminaries}

Let $F$ be a smooth curve of genus $g \geq 3$ and an let $\xi \in H^{1}\left(F, T_{F}\right)$ be a non-trivial infinitesimal deformation of $F$. To simplify notations we call rank of $\xi$ to the rank of the cup-product map

$$
H^{0}\left(F, \omega_{F}\right) \stackrel{\cdot \xi}{\longrightarrow} H^{1}\left(F, \mathcal{O}_{F}\right)
$$

considered in the Introduction. We keep the notation $W_{\xi}$ for the kernel of this map. Let $F$ be a smooth plane curve defined as the zero locus of the homogeneous polynomial $f \in \mathbb{C}[x, y, z]=S$ of degree $d$. Its Jacobian ring $R$ is the graded ring

$$
R=\bigoplus_{n \geq 0} R^{n}=\bigoplus_{n \geq 0}\left(S^{n} / J^{n}\right) .
$$

Here $J^{n}$ is the degree $n$ part of the Jacobian ideal $J=\left(f_{x}, f_{y}, f_{z}\right)$. As $F$ is smooth, $\left\{f_{x}, f_{y}, f_{z}\right\}$ is a regular sequence and this implies that $R$ satisfies the following properties:

Theorem 2.1 (Macaulay). Let $N$ be $3(d-2)$. Then $R^{N}$ has dimension 1 and, for every $k$ such that $0 \leq k \leq N$ we have that the multiplication map

$$
R^{k} \otimes R^{N-k} \rightarrow R^{N}
$$

is a perfect pairing. Moreover $R^{k}=0$ for $k>N$ or $k<0$ and the dimension of $R^{k}$ for $0 \leq k \leq N$ is determined only by $d$.

In addition to these, Griffiths proved that one can read canonically several pieces of the Hodge structure of $F$ in $R$. More precisely

Theorem 2.2. Let $R$ be the Jacobian ring of a smooth plane curve of degree $d$. Then

- $H^{0}\left(F, \omega_{F}\right) \simeq S^{d-3}=R^{d-3}$;

- $H^{1}\left(F, \mathcal{O}_{F}\right) \simeq R^{N-d+3}=R^{2 d-3}$;

- the subspace of $H^{1}\left(F, T_{F}\right)$ of all the infinitesimal deformations that preserve the planarity of $F$ is isomorphic to $R^{d}$;

- multiplication in $R$ induces, using the previous identifications, cup product of the corresponding elements.

We refer to V03 for a proof of these facts.

Next we quote two theorems of Green concerning properties of $S=\mathbb{C}[x, y, z]$. We stress that they are valid in any dimension although we state (and use) them only in dimension $n=2$. The first theorem can be found in [G94, Lecture 7, page 74], putting $p=0$ :

Theorem 2.3 (Green). Let $W$ be a subspace of $S^{a}$ of codimension $c$ and assume that $|W|$ is a base point free linear system on $\mathbb{P}^{2}$. Then, for any $m \geq c$ one has that the multiplication map

$$
W \otimes S^{m} \rightarrow S^{m+a}
$$

is surjective.

In order to state the second theorem of Green we need some notation. Given a positive integer $a$, for any $c \geq 0$ there is a unique expression

$$
c=\left(\begin{array}{c}
k_{a} \\
a
\end{array}\right)+\left(\begin{array}{c}
k_{a-1} \\
a-1
\end{array}\right)+\cdots+\left(\begin{array}{c}
k_{1} \\
1
\end{array}\right)=\left(\begin{array}{c}
k_{a} \\
a
\end{array}\right)+\left(\begin{array}{c}
k_{a-1} \\
a-1
\end{array}\right)+\cdots+\left(\begin{array}{c}
k_{\delta} \\
\delta
\end{array}\right),
$$

such that $k_{a}>k_{a-1}>\cdots>k_{\delta} \geq \delta>0$. The numbers $\left(k_{a}, k_{a-1}, \ldots, k_{\delta}\right)$ are uniquely identified by this definition and are called Macaulay's Coefficients of c with respect to a. 
If $\left(k_{a}, k_{a-1}, \ldots, k_{\delta}\right)$ are the Macaulay coefficients with respect to $a$, we denote by $c_{\langle a\rangle}$ the number

$$
c_{\langle a\rangle}=\left(\begin{array}{c}
k_{a}-1 \\
a
\end{array}\right)+\left(\begin{array}{c}
k_{a-1}-1 \\
a-1
\end{array}\right)+\cdots+\left(\begin{array}{c}
k_{\delta}-1 \\
\delta
\end{array}\right)
$$

where $\left(\begin{array}{c}m \\ n\end{array}\right)=0$ if $m<n$. Keeping this terminology we can state the following theorem:

Theorem 2.4 (Green, [G89]). Let $W \subset S^{a}$ be a linear system with codimension c. Let $H$ be a general line in $\mathbb{P}^{2}$. Then the codimension $c_{H}$ of the image of the restriction map

$$
W \longrightarrow H^{0}\left(H, \mathcal{O}_{H}(a)\right)
$$

satisfies $c_{H} \leq c_{\langle a\rangle}$.

Corollary 2.5. Under the assumption of Theorem 2.4. if $c<a$ then $c_{H}=0$, i.e. the restriction map $W \rightarrow H^{0}\left(H, \mathcal{O}_{H}(a)\right)$ is surjective.

Proof. If $c \leq a$ we can write $c=a-r$ for some $r$ such that $0 \leq r \leq a$. As

$$
c=a-r=\left(\begin{array}{l}
a \\
a
\end{array}\right)+\left(\begin{array}{l}
a-1 \\
a-1
\end{array}\right)+\cdots+\left(\begin{array}{l}
r+1 \\
r+1
\end{array}\right)
$$

we have that $(a, a-1, \ldots, r+1)$ are the Macaulay's coefficients of $c$ with respect to $a$. Therefore

$$
c_{\langle a\rangle}=\left(\begin{array}{c}
a-1 \\
a
\end{array}\right)+\left(\begin{array}{c}
a-2 \\
a-1
\end{array}\right)+\cdots+\left(\begin{array}{c}
r \\
r+1
\end{array}\right)=0 .
$$

By definition of $c_{H}$ we have that the restriction is surjective as claimed.

\section{Proof of the Theorem (1.3)}

The Theorem (1.3) in the introduction asserts that given a smooth curve $F$ of degree $d$, the rank of a non trivial infinitesimal deformation of $F$ which preserves the planarity of $F$ is bounded below by $d-3$. By using the identifications provided by Griffiths' results in (2.2) this translates into the following statement:

Theorem 3.1. Let $F$ be a smooth plane curve of degree $d \geq 5$ and let $R$ be its Jacobian ring. Let $\xi \in R^{d} \backslash\{0\}$, then the rank of the map

$$
S^{d-3}=R^{d-3} \stackrel{\cdot \xi}{\longrightarrow} R^{2 d-3}
$$

is at least $d-3$.

Proof. Denote by $W=W_{\xi} \subset S^{d-3}$ the kernel of the multiplication map

$$
\xi: S^{d-3} \rightarrow R^{2 d-3} \text {. }
$$

Note that the codimension of $W$ in $S^{d-3}$ is equal to the rank of $\xi$. The proof is divided in two cases depending of the existence or not of a fixed loci.

Case 1: $|W|$ is base-point-free.

We are going to see that $\operatorname{rk}(\xi) \geq d-2$. Assume that the opposite is true, i.e. that $\operatorname{rk}(\xi) \leq d-3$ holds. Then, by Green's Theorem, for every $m \geq \operatorname{cod}_{S^{d-3}}(W)=\operatorname{rk}(\xi)$ we have that the multiplication map

$$
\mu_{m}: W \otimes S^{m} \rightarrow S^{m+d-3}
$$

is surjective. In particular, as $\operatorname{rk}(\xi) \leq d-3$ we can take $m=d-3$. Hence we have

$$
\mu_{d-3}: W \otimes S^{d-3} \rightarrow S^{2 d-6}
$$


is surjective and the same holds for the map obtained by passing to the quotient, i.e. to the Jacobian ring:

$$
\mu_{d-3}: W \otimes R^{d-3} \rightarrow R^{2 d-6}
$$

But this is impossible since by the definition of $W$, the image of $\mu_{d-3}$ is killed by $\xi \neq 0$, hence the pairing

$$
R^{2 d-6} \otimes R^{d} \rightarrow R^{3 d-6}=R^{N}
$$

is degenerated contradicting Macaulay's Theorem. Hence we have necessarily $\operatorname{rk}(\xi) \geq d-2$ as claimed.

\section{Case 2: $|W|$ is not base-point-free.}

First we observe that we can assume that there are no base components. Indeed, assume that there exists a curve $C$ of degree $0<d^{\prime}<d-3$ in the fixed part of $|W|$. Then we have that $W \subset C \cdot S^{d-d^{\prime}-3}$ and therefore $\operatorname{dim} W \leq \operatorname{dim} S^{d-d^{\prime}-3} \leq \operatorname{dim} S^{d-4}$, hence

$$
\operatorname{codim} W \geq\left(\begin{array}{c}
d-1 \\
2
\end{array}\right)-\left(\begin{array}{c}
d-2 \\
2
\end{array}\right)=d-2
$$

as wanted.

Hence now on we assume that $|W|$ has only isolated base points. We proceed by contradiction, so assume that $\operatorname{rk}(\xi) \leq d-4$ holds.

Let $Z$ be the base locus of $|W|$ and denote by $\mathcal{I}_{Z}$ the ideal sheaf of $Z$ as subscheme of $\mathbb{P}^{2}$. Then the evaluation induces a surjection

$$
W \otimes \mathcal{O}_{\mathbb{P}^{2}} \rightarrow \mathcal{I}_{Z}(d-3) .
$$

Denoting by $M_{W}$ its kernel we have the short exact sequence

$$
0 \longrightarrow M_{W} \longrightarrow W \otimes \mathcal{O}_{\mathbb{P}^{2}} \stackrel{e v}{\longrightarrow} \mathcal{I}_{Z}(d-3) \longrightarrow 0 .
$$

Let $s$ be a general element in $S^{1}=H^{0}\left(\mathbb{P}^{2}, \mathcal{O}_{\mathbb{P}^{2}}(1)\right)$. As the base points are isolated, we can assume that the line $L=\{s=0\}$ is disjoint with $Z$. By considering the multiplication by $s$, the short exact sequence (1) induces the following commutative diagram with exact rows and columns

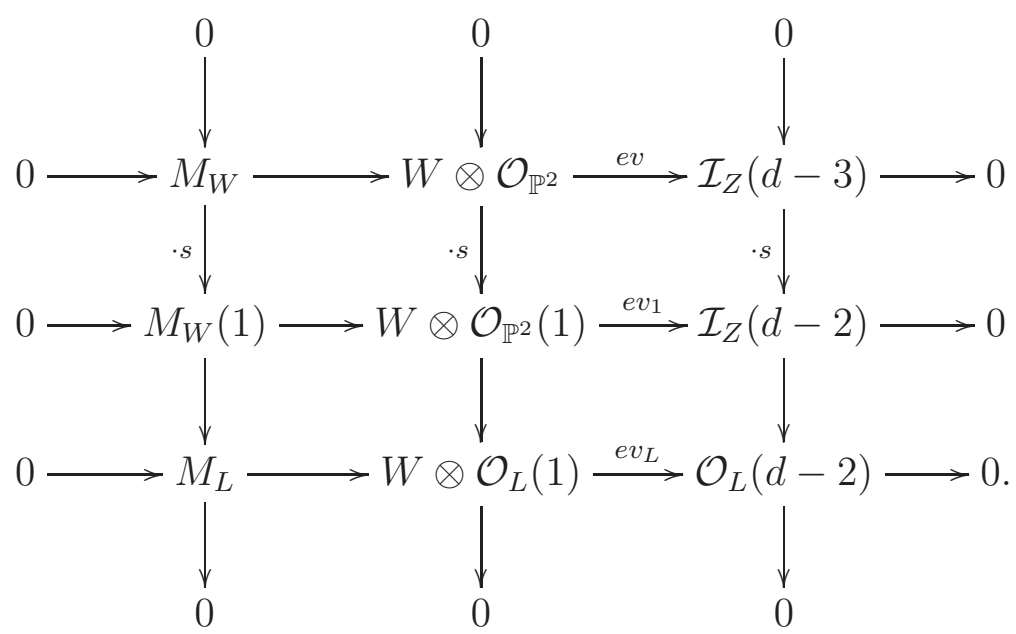

The sheaf $M_{L}$ in the third row is by the definiton the kernel of $e v_{L}$. Notice that the rightmost sheaf of the last row is $\mathcal{O}_{L}(d-2)$ because we are assuming that $L$ is disjoint from $Z$.

Claim: The vanishing $H^{1}\left(L, M_{L}\right)=0$ holds. 
Proof. (of the claim). Indeed, under our hypothesis, Corollary 2.5 implies that the restriction map

$$
W \rightarrow H^{0}\left(L, \mathcal{O}_{L}(d-3)\right)
$$

is surjective. Tensoring with $H^{0}\left(L, \mathcal{O}_{L}(1)\right)$ we get that also the evaluation map

$$
e v_{l}: W \otimes H^{0}\left(L, \mathcal{O}_{L}(1)\right) \longrightarrow H^{0}\left(L, \mathcal{O}_{L}(d-3)\right) \otimes H^{0}\left(L, \mathcal{O}_{L}(1)\right) \rightarrow H^{0}\left(L, \mathcal{O}_{L}(d-2)\right)
$$

is surjective. Then the cohomology sequence of the third row in the diagram gives the vanishing.

By taking cohomology in the second row we have a map:

$$
W \otimes S^{1} \stackrel{\eta_{1}}{\longrightarrow} H^{0}\left(\mathbb{P}^{2}, \mathcal{I}_{Z}(d-2)\right) \longrightarrow H^{1}\left(\mathbb{P}^{2}, M_{W}(1)\right) \longrightarrow 0
$$

and denote with $W_{1}$ the image of $\eta_{1}$. Our strategy is to replace $W$ by $W_{1} \subset S^{d-2}$ and apply again the same argument in order to finally reach a subspace of $W_{2} \subset S^{d-1}$ where it is easier to finish the proof as we will see below. Hence we need to show that the codimension of $W_{1}$ in $S^{d-2}$ is lower than or equal to the codimension of $W$ in $S^{d-3}$. To prove this we consider the cohomology exact sequences of the first two rows of the diagram (2):

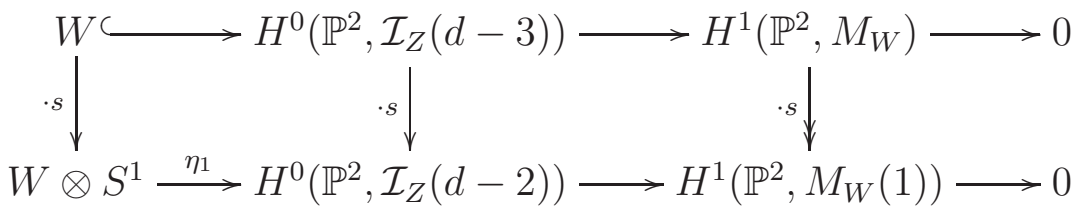

The last vertical arrow is surjective due to the claim. Therefore

$$
\operatorname{codim}_{H^{0}\left(\mathbb{P}^{2}, \mathcal{I}_{Z}(d-2)\right)} W_{1}=h^{1}\left(\mathbb{P}^{2}, M_{W}(1)\right) \leq h^{1}\left(\mathbb{P}^{2}, M_{W}\right)=\operatorname{codim}_{H^{0}\left(\mathbb{P}^{2}, \mathcal{I}_{Z}(d-3)\right)} W .
$$

Now we need to compare $\operatorname{codim}_{S^{d-2}} H^{0}\left(\mathbb{P}^{2}, \mathcal{I}_{Z}(d-2)\right)$ with $\operatorname{cod}_{S^{d-3}} H^{0}\left(\mathbb{P}^{2}, \mathcal{I}_{Z}(d-3)\right)$. As we will observe in a moment, they coincide as a consequence of the vanishing of $H^{1}\left(L, M_{L}\right)=0$.

Claim: We have the equality:

$$
\operatorname{codim}_{S^{d-2}} H^{0}\left(\mathbb{P}^{2}, \mathcal{I}_{Z}(d-2)\right)=\operatorname{codim}_{S^{d-3}} H^{0}\left(\mathbb{P}^{2}, \mathcal{I}_{Z}(d-3)\right) .
$$

Proof. (of the claim). We start again with the diagram (2). The cohomology exact sequence of the last two columns gives

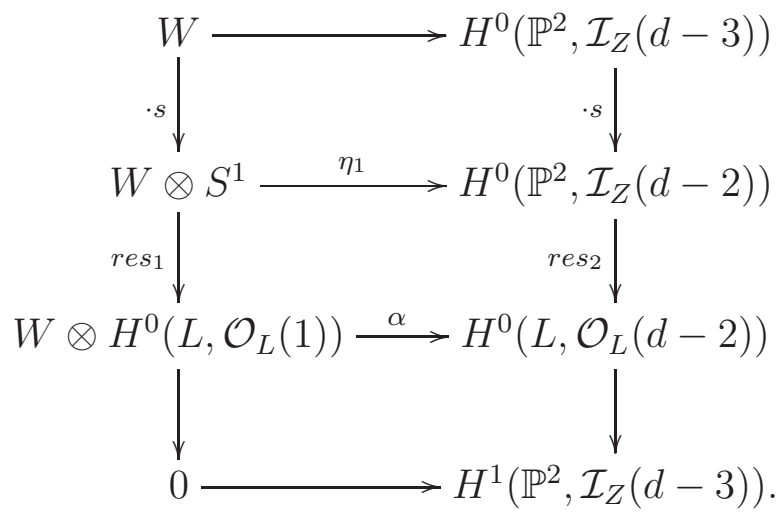


Since $H^{1}\left(L, M_{L}\right)=0$ both $\alpha$ and $\alpha \circ$ res $_{1}$ are surjective. Therefore res $_{2}$ is also surjective. This implies the isomorphism

$$
H^{1}\left(\mathbb{P}^{2}, \mathcal{I}_{Z}(d-3)\right) \stackrel{\cong}{\longrightarrow} H^{1}\left(\mathbb{P}^{2}, \mathcal{I}_{Z}(d-2)\right) .
$$

Now consider the diagram:

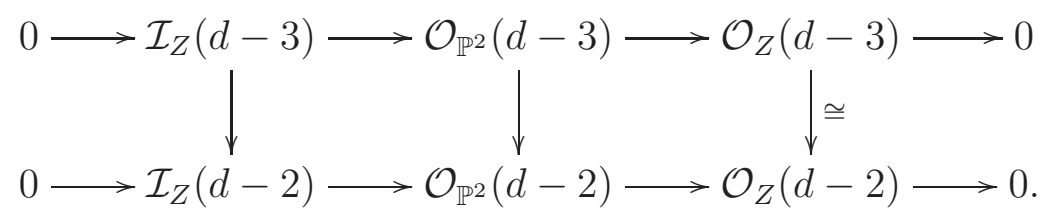

Implementing the isomorphism above we obtain the claim.

Combining the inequality (3) with the claim we have:

$$
\operatorname{cod}\left(W_{1}\right) \leq \operatorname{cod}(W) \leq d-4 .
$$

Observe that $\left|W_{1}\right|$ has, by construction, the same base locus of $|W|$ and, as we have just proven, $\operatorname{cod}\left(W_{1}\right) \leq d-4$. Hence, we can apply the same argument using $W_{1}$ instead of $W$ starting with the surjection

$$
W_{1} \otimes \mathcal{O}_{\mathbb{P}^{2}} \rightarrow \mathcal{I}_{Z}(d-2) .
$$

By doing this we obtain $W_{2} \subset S^{d-1}$ which satisfy

$$
\operatorname{cod}\left(W_{2}\right) \leq \operatorname{cod}\left(W_{1}\right) \leq \operatorname{cod}(W) \leq d-4 .
$$

To finish the proof we consider the subspace

$$
\tilde{W}:=W_{2}+\left\langle f_{x}, f_{y}, f_{z}\right\rangle=W_{2}+J^{d-1} .
$$

Observe that in $\tilde{W}$ there is a section which doesn't vanish on at least one point (we are assuming that $Z$ is not empty) of the base locus, as the partial derivatives cannot all vanish in a point. Hence the dimension of $\tilde{W}$ has increased at least by 1 and

$$
\operatorname{cod}(\tilde{W}) \leq \operatorname{cod}\left(W_{2}\right)-1 \leq d-5 .
$$

Moreover $\tilde{W}$ is base point free by construction so we can apply Green's Theorem (2.3) again with $m=d-5$ and we obtain that the multiplication map

$$
\tilde{W} \otimes S^{d-5} \rightarrow S^{d-5+d-1}=S^{2 d-6}
$$

is surjective.

Passing to the quotient by the Jacobian ideal we have a surjective map

$$
W_{2} \otimes R^{d-5} \rightarrow R^{2 d-6} .
$$

This implies by the definition of $W_{2}$ (image of $W_{1} \otimes S^{1}$ ) and $W_{1}$ (image of $W \otimes S^{1}$ ) that all the elements in $R^{2 d-6}$ are orthogonal to $\xi$ which contradicts Macaulay's Theorem. This finishes the prove of the bound.

Finally we see that the bound is sharp. Fix $d \geq 4$ and let $F$ be the Fermat curve of degree $d$ in $\mathbb{P}^{2}$, i.e. the zero locus of the polynomial $f=x^{d}+y^{d}+z^{d}$. In this case, the Jacobian ideal is simply

$$
J=\left(x^{d-1}, y^{d-1}, z^{d-1}\right)
$$

and one can easily prove that $R^{N}=\left\langle(x y z)^{d-2}\right\rangle$. Consider the element $\left[x^{d-2} y^{2}\right] \in R^{d}$ and denote it by $\xi$. Observe, moreover, that this is not zero as $x^{d-2} y^{2} \notin J$. If $m=x^{a} y^{b} z^{c}$ 
with $a+b+c=d-3$, we have $m \cdot \xi \neq 0$ if and only if $a=0$ and $0 \leq b \leq d-4$. Hence the image of the multiplication by $\xi$ is generated by the $d-3$ elements of

$$
\left\{\left[x^{d-2} y^{2+a} z^{d-3-a}\right] \mid 0 \leq a \leq d-4\right\} .
$$

As they are independent we have $\operatorname{rk}(\xi)=d-3$. Notice that $W$ is generated by monomials and $x^{d-3}, y^{d-3} \in W$ but $z^{d-3} \notin W$, hence the base locus of $|W|$ is $P_{2}=(0: 0: 1)$.

Remark 3.2. In Theorem 3.1 we have proven that the bound $\operatorname{rk}(\cdot \xi) \geq d-3$ is sharp by showing that for the Fermat curve of degree $d$ there exists an element in $R^{d}$ whose rank is exactly $d-3$. There are other curves that have this property. For example, denote by $f_{\lambda} \in S^{5}$ the polynomial $x^{5}+y^{5}+z^{5}+5 \lambda x^{3} y^{2}$ with $\lambda \in \mathbb{C}$ and consider the quintic $F_{\lambda}=\left\{f_{\lambda}=0\right\}$. Notice that $F_{0}$ is the Fermat quintic so the general quintic of this type is indeed smooth. Moreover, as $x^{3} y^{2}$ is not zero in the Jacobian ideal of the Fermat quintic, we know that $F_{\lambda}$ is not biholomorphic to $F_{0}$ for $\lambda$ general. If we denote by $R_{\lambda}$ the Jacobian ideal of $F_{\lambda}$ it is easy to see that $\xi_{\lambda}=\left[x^{3} y^{2}\right]$ is not zero in $R_{\lambda}$. Now we want to prove that $\operatorname{rk}\left(\xi_{\lambda}\right)=2$ for all $\lambda \in \mathbb{C}$. As we have already seen, $\xi_{\lambda}$ is non trivial so, by Theorem 1.3 we know that $\operatorname{rk}\left(\xi_{\lambda}\right) \geq 2$. In order to see that the equality holds it is enough to prove that $\operatorname{dim} W_{\xi_{\lambda}} \geq 4$ (as $\operatorname{dim} R_{\lambda}^{2}=6$ ). With a little bit of effort one can show that

$$
x^{2}, x y, y^{2}, x z+18 \lambda^{3} y z \in W_{\xi_{\lambda}}
$$

thus proving the claim.

\section{REFERENCES}

[AP16] Albano A., Pirola G. P. Dihedral monodromy and Xiao fibrations Ann. Mat. Pura Appl. (4) 195 (2016), no. 4, 1255-1268.

[BGN16] Barja M. A., Gonzalez-Alonso V., Naranjo, J. C. Xiao's conjecture for general fibred surfaces To appear in Journal für die reine und angewandte Mathematik (DOI 10.515/Crelle-2015-0080).

[C95] Cai J.-X., Irregularity of certain algebraic fiber spaces, Manuscripta Math. 95 (1998), no. 3, 273-287.

[G89] M. Green, Restrictions of linear series to hyperplanes, and some results of Macaulay and Gotzmann, Algebraic curves and projective geometry (Trento, 1988), 76-86, Lecture Notes in Math., 1389, Springer, Berlin, 1989.

[G94] M. Green, Infinitesimal methods in Hodge theory, Algebraic cycles and Hodge theory (Torino, 1993), 1-92, Lecture Notes in Math., 1594, Springer, Berlin, 1994.

[P92] Pirola G. P. On a conjecture of Xiao J. Reine Angew. Math. 431 (1992), 75-89.

[X87] Xiao G. Fibered algebraic surfaces with low slope Math. Ann. 276 (1987), 449-466.

[X88] Xiao G. Problem list, in: Birational geometry of algebraic varieties: Open problems (XXIII International Symposium), Division of Mathematics, Taniguchi Foundation (1988), 36-40.

[V03] Voisin, C. Hodge theory and complex algebraic geometry. II, Cambridge Studies in Advanced Mathematics, 77. Cambridge University Press, Cambridge, 2003.

(Filippo F. Favale) Department of Mathematics, Università degli Studi di Trento, Via Sommarive 14, 38123 Trento, ItAly

E-mail address: filippo.favale@unitn.it

(Juan Carlos Naranjo) Departament de Matemàtiques i InformàticA, Universitat de Barcelona, Gran Via 585, 08007 Barcelona, Spain

E-mail address: jcnaranjo@ub.edu

(Gian Pietro Pirola) Department of Mathematics, Università degli Studi di Pavia, Via Ferrata 1, 27100 Pavia, Italia

E-mail address: gianpietro.pirola@unipv.it 